\title{
Antipsychotic Induced Malignant Syndrome: A Native Inpatient Evaluation
}

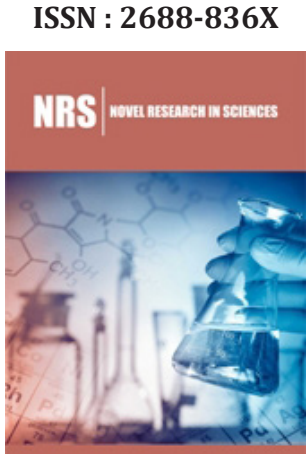

*Corresponding author: Shafti SS, University of Social Welfare and Rehabilitation Sciences (USWR), Razi Psychiatric Hospital, P.O. Box 18735-569, Tehran, Iran

Submission: 眥 September 04, 2019

Published: 䟧 September 11,2019

Volume 2 - Issue 1

How to cite this article: Shafti SS, Memarie A, Rezaie M and Hamidi M. Antipsychotic Induced Malignant Syndrome: A Native Inpatient Evaluation. Nov Res Sci.2(1). NRS.000527.2019.

DOI: 10.31031/NRS.2019.2.000527

Copyright@ Shafti SS, This article is distributed under the terms of the Creative Commons Attribution 4.0 International License, which permits unrestricted use and redistribution provided that the original author and source are credited.

\author{
Shafti SS*, Memarie A, Rezaie M and Hamidi M
}

Full Professor of Psychiatry, Iran

\begin{abstract}
Introduction: Neuroleptic malignant syndrome (NMS) is a life-threatening complication that can occur anytime during antipsychotic treatment. In the present assessment the incidence and clinical profile of NMS have been probed among a sample of non-western psychiatric inpatients and compared with the available data in literature regarding prevalence and other associated clinical physiognomies.
\end{abstract}

Methods: As a retrospective, record based evaluation, all cases that had been diagnosed absolutely as NMS during the last sixty-two months, after ruling out other imaginable differential diagnosis, like encephalitis, meningitis and serotonin syndrome, had been included in the present investigation. Clinical diagnosis, as well, was based on Diagnostic and Statistical Manual of Mental Disorders, $5^{\text {th }}$ edition (DSM5). Appraisal of independent variables had been analyzed by means of Compression of proportions. Statistical significance as well, had been defined as $\mathrm{p}$ value $\leq 0.05$.

Results: Among 19814 psychiatric patients hospitalized in razi psychiatric hospital, during a sixty-two months' period (April of 2013-August 2018), eighteen cases ( $\mathrm{N}=18$ ) had been referred to Intensive Care Unit (ICU) with diagnosis of NMS. The most prevalent symptom was fever, which was present in $100 \%$ of cases and was significantly more frequent in comparison with. No significant difference was evident between the remaining symptoms as regards incidence. Also, there was not any significant difference between the first generation versus second generation antipsychotics. NMS was significantly more prevalent among patients suffering from schizophrenia, and amongst male patients. Similarly, it was significantly more widespread amid 18-65 years old age-group, especially age group of 30-39. Fatality was limited to only one patient.

Conclusion: While no significant difference was evident between first generation as opposed to second generation antipsychotics, NMS was significantly more prevalent among young and male patients suffering from schizophrenia.

Keywords: Neuroleptic malignant syndrome; Typical antipsychotic; Atypical antipsychotic; Schizophrenia

\section{Introduction}

Neuroleptic malignant syndrome (NMS) is a life-threatening complication that can occur anytime during antipsychotic treatment [1]. The motor and behavioral symptoms include muscular rigidity and dystonia, akinesia, mutism, obtundation, and agitation. The autonomic symptoms include hyperthermia, diaphoresis, and increased pulse and blood pressure [2]. Laboratory findings include an increased white blood cell count and increased levels of creatinine phosphokinase, liver enzymes, plasma myoglobin, and myoglobinuria, occasionally associated with renal failure [3]. These complications typically occur within days after exposure to an antipsychotic. NMS, however, can vary in terms of symptom presentation, progression, and outcome [3]. A hypodopaminergic state which interferes with temperature regulation is the postulated mechanism for the development of this syndrome [1].

NMS is associated with dopamine antagonists, the abrupt discontinuation of antiparkinsonian medications and, rarely, the abrupt discontinuation of a dopaminergic agent. This suggests that fluctuation in dopamine binding may be important in its etiology [2]. Because dopamine receptors are widely distributed in the CNS and spinal cord, it is likely that the various symptoms seen in NMS are accounted for by dopamine dysregulation in different areas [2]. Abnormal calcium availability in muscle cells may also contribute, given the potential for muscle damage reflected by high creatine kinase elevations [3]. The occasional familial clustering of NMS leads to an analysis of genetic factors which may predispose to this condition [4]. For example, the A1 allele of the dopamine D2 receptor gene was observed to 
be overrepresented in patients who develop NMS compared with those who do not [4]. This allele reduces the density and function of the dopamine receptor in the striatum and caudate nuclei, leading to reduced dopaminergic metabolism in those CNS areas with high concentrations of the neurotransmitter. NMS can be fatal or produce residual sequelae (e.g., cognitive or other neurological deficits) [4]. Therefore, management involves the aggressive use of supportive measures, as well as more specific interventions.

Early identification is important and patients at higher risk should be carefully monitored [4]. One easily treatable risk factor is dehydration. In addition, patients should avoid excessively high temperatures [2]. Very mild cases of low-grade temperature increase or mild creatine phosphokinase (CPK) elevations may be treated in a psychiatric unit, while a rapid onset or worsening of symptoms requires transfer to a medical intensive care unit [5]. The relative risk of developing NMS between the two generations of antipsychotics, however, is difficult to compare [5,6]. About 0.01 to 0.02 percent of patients treated with antipsychotics develop neuroleptic malignant syndrome. Men are affected more frequently than women, and young patients are affected more commonly than elderly patients. The mortality rate can reach 10 to 20 percent or even higher when depot antipsychotic medications are involved [7]. The diagnosis is often missed in the early stages, and the withdrawal or agitation may mistakenly be considered to reflect an exacerbation of the psychosis [8]. In the present assessment the incidence and clinical profile of NMS have been probed among a great sample of non-western psychiatric inpatients and compared with the available data in literature regarding prevalence and other associated clinical physiognomies.

\section{Methods}

Razi psychiatric hospital in south of capital city of Tehran, as one of the largest and oldest public psychiatric hospitals in the Middle East, which has been established formally in 1917 and with a capacity around 1375 active beds, had been selected as the field of study in the present retrospective assessment. For evaluation, all cases that had been diagnosed absolutely as NMS during the last sixty-two months, after ruling out other imaginable differential diagnosis, like encephalitis, meningitis and serotonin syndrome, had been included in the present investigation. Clinical diagnosis, as well, was based on Diagnostic and Statistical Manual of Mental Disorders, $5^{\text {th }}$ edition (DSM-5) [9].

\section{Statistical analysis}

Appraisal of independent variables had been analyzed by means of 'comparison of proportions. Statistical significance as well, had been defined as $p$ value $\leq 0.05$. MedCalc Statistical Software version 15.2 was used as statistical software tool for analysis.

\section{Result}

As said by results, among 19814 psychiatric patients hospitalized in razi psychiatric hospital, during a sixty-two months' period (April of 2013- August 2018), eighteen cases ( $N=18$ ) had been referred to Intensive Care Unit (ICU) with diagnosis of NMS (annual incidence $=0.017 \%$ ). The mean total days between admission in the psychiatric ward up to transfer to the ICU was around $3.05 \pm 2.54$ days, and the mean total days of treatment as regards NMS, before returning to the psychiatric ward was around $7.83 \pm 6.11$ days. The most prevalent symptom was fever, which was present in $100 \%$ of cases (mean total average $=39.71 \pm 0.58$ ) and was significantly more frequent in comparison with other ones $(\mathrm{z}=2.68, \mathrm{p}<0.007, \mathrm{CI}$ $95 \%=0.089,0.576$ ) (Table 1). No significant difference was evident between the remaining symptoms as regards incidence. Concerning contrast between prescribed typical antipsychotics [haloperidol $(n=9)$, chlorpromazine $(n=1)$, thioridazine $(n=1)]$ and atypical antipsychotics [olanzapine $(n=4)$, risperidone $(n=3)]$, 'Compression of proportions' did not show any significant difference between first generation as opposed to second generation antipsychotics $(\mathrm{z}=1.33$, $\mathrm{p}<0.18$, CI 95\%=-0.10,0.54). While not a significant finding, around thirty-eight percent $(\mathrm{n}=7)$ of patients had received parenteral antipsychotic (haloperidol, IM or IV), which consisted around $70 \%$ of all cases that had received only that kind of typical antipsychotic. But, consistent with analysis, NMS was significantly more prevalent among patients suffering from schizophrenia $(n=12)$, in comparison with bipolar disorder $(\mathrm{n}=6)(\mathrm{z}=2.00, \mathrm{p}<0.04, \mathrm{CI} 95 \%=0.006,0.66)$. Moreover, it was significantly more frequent amongst male patients $(n=13)$, in comparison with female cases $(n=5)(z=2.66, p<0.007$, CI $95 \%=0.11,0.77)$. Similarly, it was significantly more widespread amid 18-65 years old age-group $(\mathrm{n}=15,83.33 \%)$, in comparison with adolescent ( $\leq 18$ years old; $n=2,11.11 \%$ ) or elderly ( $\geq 65$ years old; $\mathrm{n}=1,5.55 \%)$ patients $(\mathrm{z}=4.00, \mathrm{p}<0.0001$, C I 95\%=0.34, 0.99). The highest risk of NMS was evident in the age group of 30-39 ( $n=8$, 44.44\%), in comparison with 20-29 ( $n=3,16.6 \%)$ and 40-49 $(n=4$, $22.22 \%$ ). Mortality was limited to merely one patient after thirty days' treatment in ICU (annual incidence=0.0009\%).

Table 1: Prevalence of NMS symptoms.

\begin{tabular}{|c|c|c|}
\hline Symptoms & Number & Percentage \\
\hline Fever & 18 & $100 \%$ \\
\hline Perspiration & 12 & $66 \%$ \\
\hline Leukocytosis & 11 & $61 \%$ \\
\hline Disturbance of consciousness & 11 & $61 \%$ \\
\hline Blood pressure instability & 11 & $61 \%$ \\
\hline Muscle rigidity & 11 & $61 \%$ \\
\hline Liver enzymes elevations & 11 & $61 \%$ \\
\hline Increase of CPK & 9 & $50 \%$ \\
\hline Muscle sore & 9 & $50 \%$ \\
\hline
\end{tabular}

\section{Discussion}

Neuroleptic Malignant Syndrome (NMS) is a life-threatening complication of antipsychotic therapy. It is a rare but potentially fatal disorder. On the other hand, while It is often assumed to be rare, Observations suggest that rather than overestimating its frequency, we are more likely to underestimate it [10]. The diagnosis of NMS often presents a challenge because several medical conditions generate similar symptoms. Although less common now than in the past, thanks to greater awareness, it remains a risk in susceptible patients receiving conventional or atypical neuroleptics. Reducing the risk factors, early recognition of suspected cases and prompt 
management can significantly reduce morbidity and mortality of this dangerous condition. Collaboration between psychiatry and other medical specialties may be the key to a successful outcome [10]. While NMS is a diagnosis of exclusion, we should keep in mind that there is great variability of its clinical presentation in order to not exclude too quickly this syndrome in a patient treated by neuroleptic [6].

Also, while for NMS associated with atypical antipsychotic drugs, the mortality rate was lower than that with conventional antipsychotic drugs, the mortality rate may simply reflect physicians' awareness and ensuing early treatment [11]. The diagnosis of NMS commonly requires core symptoms of hyperthermia and muscle rigidity. Although diagnostic criteria for NMS have been established and are widely accepted and used, it should be recognized that atypical presentations pose a diagnostic dilemma, as hyperthermia and/or muscle rigidity may be absent or develop slowly over several days, leading to impairment or a significant delay in diagnosis and treatment [12]. Evidence from case reports and retrospective evaluations supports a concept of atypical NMS, particularly with regard to treatment with atypical antipsychotics. However, it remains unclear whether these atypical presentations represent early or impending NMS. Furthermore, it is unclear whether dysfunction in other neurotransmitter systems, in addition to dopamine, may be involved in the pathogenesis of NMS induced by atypical antipsychotics.

In patients receiving any antipsychotic, clinicians should carefully evaluate any features of NMS and should not prematurely exclude a diagnosis of NMS in cases where severe rigidity or hyperthermia is not initially apparent [12]. According to the findings of the present assessment, while the incidence of NMS in the present survey was comparable to the outcomes of Janno et al. [7], it was very lower than conclusions of Khouri et al. [6]. Nevertheless, due to lack of studies regarding the prevalence of NMS, maybe comparison of its incidence could not be a practical judgment. The same conclusion, as well, was applicable regarding the incidence of NMS' mortality, which was remarkably lower than the findings of Janno et al. [7]. Anyway, it should not be overlooked that though NMS is one of the few life-threatening psychotropic-induced complications, its infrequent occurrence and severe nature make controlled studies impossible. Maintaining a high index of suspicion and commencement of necessary medical care as soon as possible can be acknowledged as the most favorable preventive measures against NMS' mortality or complications. Maybe, availability of medical ward and ICU in psychiatric facility can shorten the interval between suspecting or detecting NMS and beginning of necessary managements, an advantage which is accessible in the present hospital and have shortened the transferal of suspicious cases from psychiatric districts to medical zone to less than a few days. Also, there was no significant difference between typical and atypical antipsychotics with regard to instigating NMS, a finding which was reasonably in congruence with the results of [6,11,13-15]. But in the current survey, and in contrary to [14], while tetrad of fever, rigidity, autonomic instability, and altered mental status have been identified as diagnostic criteria for NMS, no atypical presentation of
NMS that lack one or more characteristic features was meaningfully evident due to atypical antipsychotics. Nonetheless, while his suggestion was regarding atypical NMS precipitated by clozapine and quetiapine overdoses, in the present appraisal NMS had been originated only by standard dosages of olanzapine and risperidone, which may implicate different symptomatology in comparison with the above medications. Besides, our conclusion is somewhat in accord with Trollor et al. [13], who believes that NMS associated with atypical antipsychotic drugs manifests in a typical manner, except that clozapine-induced NMS, which appears less likely to manifest with extrapyramidal features, including rigidity and tremor.

Also, since the only adolescent in the present assessment had been prescribed olanzapine, so again our result is to some extent in agreement with the inference of Croarkin et al. [14], who had identified that Young patients can develop NMS during treatment with atypical antipsychotics and its symptoms are consistent with those described in adults. In any case, it must be emphasized that routine checking and trending of serum CPK in patients on antipsychotic drugs who present with features of NMS is recommended to facilitate diagnosis. Furthermore, in the present evaluation, non-catatonic schizophrenia, in comparison with bipolar disorder, was significantly more connected with NMS, a finding which is not in synchronization with the findings of Modi et al. [2] and Tse et al. [4], who had indicated catatonia and mood disorders as factors which may increase the risk of developing NMS.

Regarding sexual category and time of life our outcomes are comparable with the conclusions of Janno et al. [7], who had indicated that men are affected more frequently than women, and young patients are affected more commonly than elderly patients. In the present assessment the highest risk of NMS was evident in the third decade of life, and it was almost 2.5 times more among male patients in comparison with female patients. Limited period of study, due to imperfect registration and documents in the last decades, and consequently inadequate number of cases with diagnosis of NMS, as accessible samples, despite magnitude of the field of study, do not permit generalization of the findings to more than a pilot study. No doubt, further methodical studies in future will enhance our clinical conception regarding diagnosis and management of this hazardous complication of antipsychotics.

\section{Conclusion}

While no significant difference was evident between first generation as opposed to second generation antipsychotics, NMS was significantly more prevalent among young and male patients suffering from schizophrenia.

\section{References}

1. Berman BD (2011) Neuroleptic malignant syndrome: A review for neuro hospitalists. Neurohospitalist 1(1): 41-47.

2. Modi S, Dharaiya D, Schultz L, Varelas P (2016) Neuroleptic malignant syndrome: Complications, outcomes, and mortality. Neurocrit Care 24(1): 97-103.

3. Rajamani B, Kumar Y, Rahman SM (2016) Neuroleptic malignant syndrome. J Family Med Prim Care 5(1): 178-180. 
4. Tse L, Barr AM, Scarapicchia V, Rodriguez FV (2015) Neuroleptic malignant syndrome: A review from a clinically oriented perspective. Curr Neuropharmacol 13(3): 395-406.

5. Atsariyasing W, Goldman M (2014) Management of medication-related adverse effects. In: Janicak PG, Marder SR, Tandon R, et al. (Eds.) Schizophrenia: Recent Advances in Diagnosis and Treatment, Springer, New York, pp. 225-262.

6. Khouri C, Planès S, Logerot S, Villier C, Mallaret M (2016) [Case report: Neuroleptic malignant syndrome and diagnostic difficulties]. Encephale 42(3): 277-280.

7. Janno S, Holi M, Tuisku K, Wahlbeck K (2004) Prevalence of neurolepticinduced movement disorders in chronic schizophrenic inpatients. Am J Psychiatry 161(1): 160-163.

8. Zarrouf FA, Bhanot V (2007) Neuroleptic malignant syndrome: Don't let your guard down yet. Curr Psychiatry 6(8): 89-95.

9. (2013) Diagnostic and statistical manual of mental disorders. ( $\left.5^{\text {th }} \mathrm{edn}\right)$, DC: American Psychiatric Association, Washington, USA, pp. 123-132.
10. Velamoor R (2017) Neuroleptic malignant syndrome: A neuropsychiatric emergency: Recognition, prevention, and management. Asian J Psychiatr 29: 106-109.

11. Ananth J, Parameswaran S, Gunatilake S, Burgoyne K, Sidhom T (2004) Neuroleptic malignant syndrome and atypical antipsychotic drugs. J Clin Psychiatry 65(4): 464-470.

12. Picard LS, Lindsay S, Strawn JR, Kaneria RM, Patel NC, et al. (2008) Atypical neuroleptic malignant syndrome: Diagnostic controversies and considerations. Pharmacotherapy 28(4): 530-535.

13. Trollor JN, Chen X, Sachdev PS (2009) Neuroleptic malignant syndrome associated with atypical antipsychotic drugs. CNS Drugs 23(6): 477-492.

14. Croarkin PE, Emslie GJ, Mayes TL (2008) Neuroleptic malignant syndrome associated with atypical antipsychotics in pediatric patients: a review of published cases. J Clin Psychiatry 69(7): 1157-1165.

15. Teo DCL, Wong HK, Tan SN (2018) Atypical neuroleptic malignant syndrome precipitated by clozapine and quetiapine overdose: a diagnostic challenge. Innov Clin Neurosci 15(7-8): 20-22. 